\title{
Implementation of artificial intelligence in optimisation of technological processes
}

\author{
Jerzy Lipski ${ }^{1, *}$, and Kazimierz Zaleski ${ }^{2}$ \\ ${ }^{1}$ Lublin University of Technology, Management Faculty, Poland \\ ${ }^{2}$ Lublin University of Technology, Mechanical Engineering Faculty, Poland
}

\begin{abstract}
This article introduces an algorithm for determining optimal parameters of a technological process. The objective function is the processing time of operations (efficiency) at the constraint of quality requirements of finish according to the designer specification. The problem of selecting a correct combination of processing parameters may only be solved when the cause-andeffect relationship between the finish quality and the machining settings is known. If the process considered for optimisation is repeatable, it appears economically viable to invest resources in the development of a model that would describe these relationships. To this end, we propose employing the artificial neural network trained on the progressions obtained from the tests. In the second stage, the Multiple-Input-Multiple-Output (MIMO) system, capable of representing relationships of nonlinear nature, was implemented for the optimisation of the objective function. The paper presents the application of the developed algorithm in determination of optimal parameters for the roller burnishing process of surface treatment. A technologist/software user defines the range of acceptable surface finishes. The optimisation algorithm determines a set of modifiable parameters that ensure minimal processing time at a specified surface finish requirements constraint.
\end{abstract}

\section{Introduction}

The technologies at the disposal of modern production companies are highly customisable. Parameters of a designed process are modified to obtain the desired final product. However, the aim must be realised at maximum efficiency of workstations processing the technological jobs.

It is particularly important to realise the defined objective function for the lot or mass production, as the loss of even a single piece in a batch of thousand constitutes a serious blow to the efficiency of production, which consequently translates to impeding the competitiveness of a given enterprise in the market.

The degree of complexity of utilised technologies is high and ever-increasing, therefore product parameters are related by non-linear functional relations with modifiable parameters. Furthermore, apart from the modifiable parameters, the process is affected by the individual characteristics of the tool, the technological machine or the material from which the product is made. The latter are by nature non-modifiable, and difficult to identify.

Conventional solutions to the presented problem usually involve the combination of the trial-and-error method in the form of test runs, and the experience and knowledge of the technologist or operator of the machine processing the job. Deterministic models based on the description of physical processes occurring in the realisation of the technological process may prove to resolve the issues to a certain extent.

However, practice shows that the implementation of thus determined activities proves both time-consuming and involves substantial cost, simultaneously not guaranteeing the satisfactory efficiency of the production process.

An alternative solution, which is the subject of this paper, is based on artificial neural networks and genetic algorithms. These methods are established on the data derived from the process under optimisation, hence the first step of the developed methodology consists in collection of data for analysis. To this end, the technological process should be conducted for several dozen repetitions in a range of modifiable parameter configurations, and its results registered. The second step is the development of the mathematical model of the process under investigation. However, due to the complexity of modern industrial technologies it is considerably difficult to build the conventional model accounting for the character of functional relations (the non-parametric model). Another problem faced by engineers is determining the parameters and therefore making the model functional. Some of the successful solutions have been based on multiple-parametric and non-linear models built with the implementation of artificial neural networks [1,2,3].

The neural network trains on data (sequences), obtained from the experiments. The trained network

* Corresponding author: j.lipski@pollub.pl 
provides a simulation of processes that occur in the modelled technological process $[4,5]$.

The third step consists in employing the properties of the trained network (the virtual process model) to establish the optimal set of technological parameters of the process, considering the design specifications and the maximal efficiency objective constraints. In an attempt to solve this problem it was resolved that the genetic algorithm would provide by far the most superior solution.

The steps described in the preceding paragraphs are detailed in the parts to follow.

\section{The algorithm for establishing the optimal technological process parameters}

The algorithm determining the optimal modifiable parameter configuration of the considered process consists of three steps:

- determining the input and output parameters of the modelled process; registering the values during operation of the workstation in the conditions representing batch production,

- building the virtual model of input-output functional relations in the artificial neural network;

- determining the input parameters that fulfil the defined specifications and provide optimal process efficiency in given conditions.

\subsection{Data acquisition for the neural network model}

Acquisition of data for the training of the neural network should be preceded by the analysis of acceptable ranges of determined input parameters. The potential limitations are dictated by such factors as: technical capabilities of the technological machine, the safety considerations of the process, or tool life.

The engineer must also resolve the question regarding the range of values for particular parameters, i.e. accuracy: the higher the number of designed elements in the training sets is, the higher the accuracy of the artificial neural network model in representing the characteristics of the process will be. It ought to be reminded, however, that this change will entail an increase in the number of test runs, and will therefore extend the implementation time [6].

In order to ensure that the data acquisition step runs smoothly and is adequately accurate, the workstation should be equipped with an automated and computer controlled input and output data acquisition system. The system should furthermore be capable of realising the programmed plan of modifiable input and output parameter settings of the process [6].

\subsection{Building the model of the technological process in the artificial neural network}

The artificial neural network is a digital representation of analogue networks composed of neurons, which are responsible for learning and generalisation of knowledge in living organisms. A cybernetic model of a neuron is presented in Fig. 1.

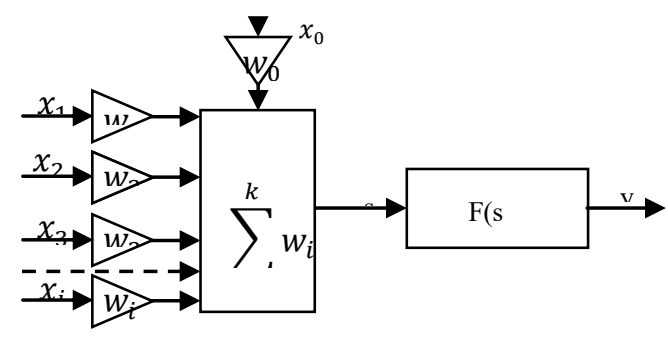

Fig.1. Model of neuron.

The studies into the mechanisms of biological nervous cells have led to developing the cybernetic model of the cell, shown in Fig. 1, which has been utilised to build the artificial neural network. The model consists of $n$ number of elements in input, which receive signals $x_{i}$ that are multiplied by weights $w_{j}$. The weighted inputs are diverse and depend on the relevance of particular inputs for outputs. The signal value is established as $x_{0}=1$, and the weighted values are set to $w_{0}$, (bias). The remaining weighted values are determined in the learning process. The obtained products are summed. The signal of the sum of products constitutes the input signal as an activation function argument $\mathrm{f}(s)$. The function generates the output signal from neuron $y$. There is a range of neuron models, which show differences regarding the application and character of activation function character.

The developed model designed to represent the actual real object is created when the separate neurons are connected to form the network [7]. In such a network we may distinguish the layer of input neurons, the hidden layers and the output layer. In the network, the output of each neuron is connected with the inputs of all neurons of the subsequent layer. The model of the network is presented in Fig. 2.

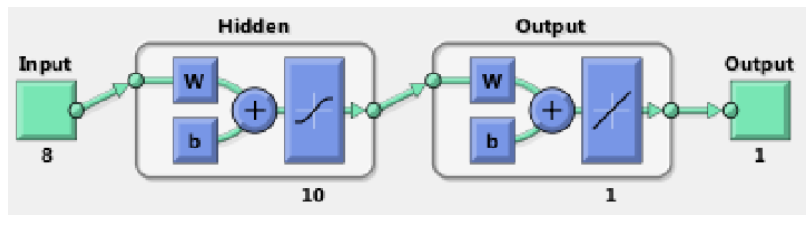

Fig.2. Artificial neural network model.

The artificial neural network is utilised in two states: trading and simulation [8]. „Deep learning” is a branch of machine learning that teaches computers to do what comes naturally to humans: learn from experience. Machine learning algorithms use computational methods to "learn" information directly from data without relying on a predetermined equation as a model. Deep learning is especially suited for image recognition, which is important for solving problems such as facial recognition, motion detection, and many advanced driver assistance technologies such as autonomous driving, lane detection, pedestrian detection, and autonomous parking.

Neural Network Toolbox ${ }^{\mathrm{TM}}$ provides simple MATLAB ${ }^{\circledR}$ commands for creating and interconnecting the layers of a deep neural network. Function $\operatorname{sim}()$ in this toolbox is usually called implicitly by calling the neural network as a function.[9] 


\subsection{Determination of the optimal parameters of the technological process}

This step of the neural network design process is aimed at determining, in the multidimensional space of input parameters, the subsets that fulfil the requirements specified in the design documentation of the product [10]. When implemented in practice, these parameters should ensure that the objectives of the technological process are achieved, at specified tolerance limits and with a view to achieving a more accurate fit with the target output. Such a strategy does not enforce searching for one solution that would fit the minimal or maximal value of the objective function. Our approach establishes additional criterion - for the selection of optimal parameter values from the viewpoint of maximal efficiency of the workstation, i.e. from the perspective of manufacturing economy. To this end, one process parameter that shows the most essential affiliation with the economy of the process should be specified, and from the determined sets it is the one that ensures the highest efficiency should be selected.

The presented algorithm proposes novel application of the virtual multiple-parametric model built in the artificial neural network, which has been traditionally applied for predicting the outputs based on the knowledge of input parameters of the technological process. The introduction of an additional algorithm allows us to establish a formerly unknown configuration of parameters which, when implemented in the technological process, ensures satisfactory quality and optimal manufacturing process efficiency.

The practical problem encountered in modelling different technological processes regarded the scenario in which two or more output parameters were to be simultaneously attained. Modelling under such constraints required employing an algorithm that would generate different sets of input parameters for each output parameter. The solution involved spreading the tolerance limits for input parameters and establishing a common subset of input parameters. By application of the efficiency constraint to the elements of this subset we obtain the set of desired process parameters.

The strategy for the determination of optimal technological parameters described in the previous paragraph was realised by means of Genetic Algorithm and Direct Search Toolbox MATLAB ${ }^{\circledR}$

\section{The determination of the roller burnishing process parameters - example}

The technological process of roller burnishing is applied with a view to decreasing the surface roughness and increasing the strain hardening of the material. The two characteristics of materials in question play a crucial role in machine parts and elements subject to cyclic local or linear pressures generated by other elements of the structure. These interactions are typically found in rolling bearings, where steel rolling elements (balls or rollers) exert such a pressure on the ring. The rolling bearing manufacturing process is realised in the large-lot production, for which reason the configuration of roller burnishing parameters should ensure that the finished product obtained from a given production cycle exhibits the target features: surface roughness and strain hardening. Both these characteristics must be within the tolerance limit specified by the designers in order for the product to attain its predesigned durability and quality.

Post-operative quality control of the surface roughness is relatively uncomplicated, particularly compared with the evaluation of strain hardening, which proves significantly more complex and time-consuming.

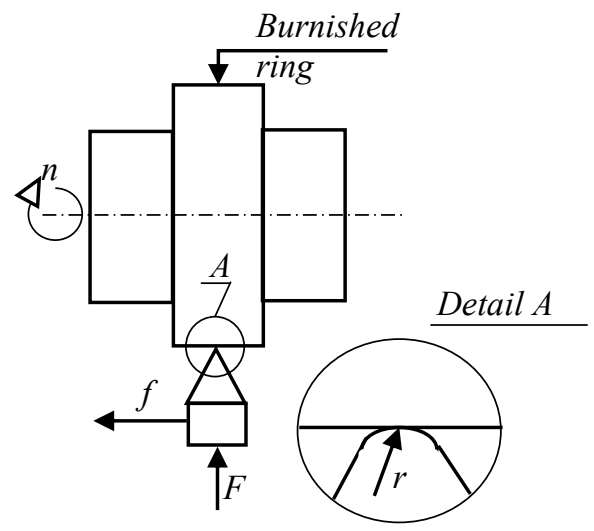

Fig.3. Kinematic structure of roller burnishing process. $n$ [rev./min] - ring revolutions, $f[\mathrm{~mm} / \mathrm{rev}]-$. feed $F[\mathrm{~N}]-$ burnishing force, $r$ [mm] - roller contour radius.

Following the preparatory operations, we carried out the procedure of selecting optimal parameters for the process of roller burnishing of rings at the target surface roughness and strain hardening constraints, observing the tolerance limits and maximal workstation efficiency. The kinematic structure of the process is shown in Fig. 3.

The first stage consisted in registering the surface roughness and hardness obtained from the 80 combinations of input parameters. The test samples were steel rings made of $100 \mathrm{Cr} 6$ ball and roller bearing steel.

Test sample dimensions were the following: outer diameter $-56 \mathrm{~mm}$, bore diameter $-55 \mathrm{~mm}$, raceway width $-10 \mathrm{~mm}$. The burnishing was applied on the outer surface of bearings, pre-treated by means of grinding (surface roughness of the pre-treated surface $\mathrm{Ra}=0.61 \div$ $0.98 \mu \mathrm{m})$. The hardness of test samples was in the range of $62 \div 64$ HRC

The burnishing operation was conducted with the multi-purpose turning lathe. The samples were attached in the spindle, fixed in the lathe centres. The burnishing tool consisted of the body and the spherical roller head made of synthetic diamond material. The tool was fixed in a special attachment that applied the desired normal load on the workpiece. The modifiable parameters of the burnishing process were: roller contour radius $r[\mathrm{~mm}]$, feed $f[\mathrm{~mm} / \mathrm{rev}$.], burnishing force $F[N]$, whereas the fixed parameter was burnishing velocity of $44 \mathrm{~m} / \mathrm{min}$. The lubrication oil dispensed during burnishing was HIPOL GL4. The factors under analysis were the surface roughness following burnishing $R a[\mu \mathrm{m}]$ and the strain hardening of the surface layer $e[\%]$. Surface roughness parameters were measured by means of Surtronic $3+$ profilometer, provided by Taylor Hobson. The 
microhardness evaluation of test samples was carried out at the loading of $0.49 \mathrm{~N}$, with the LM700AT Microindentation Hardness Tester, produced by LECO. The conducted microhardness tests have allowed us to determine the degree of strain hardening of the test sample surface layer, from the following formula:

$$
e=100 \frac{H V_{\max }-H V_{0}}{H V_{0}}, \%
$$

where: $H V_{\max }$ - maximum microhardness,

$H V_{0}$ - microhardness of material core.

The data obtained from the experimental stage of the study consisted of 803 -element input vectors and 2 output vectors. The data were divided into the train set, the validation sequence subset and the test subset.

The neural network structure was designed and trained by means of MATLAB ${ }^{\circledR}$ Neural Network Toolbox. The neural network training report is shown in Fig. 4.

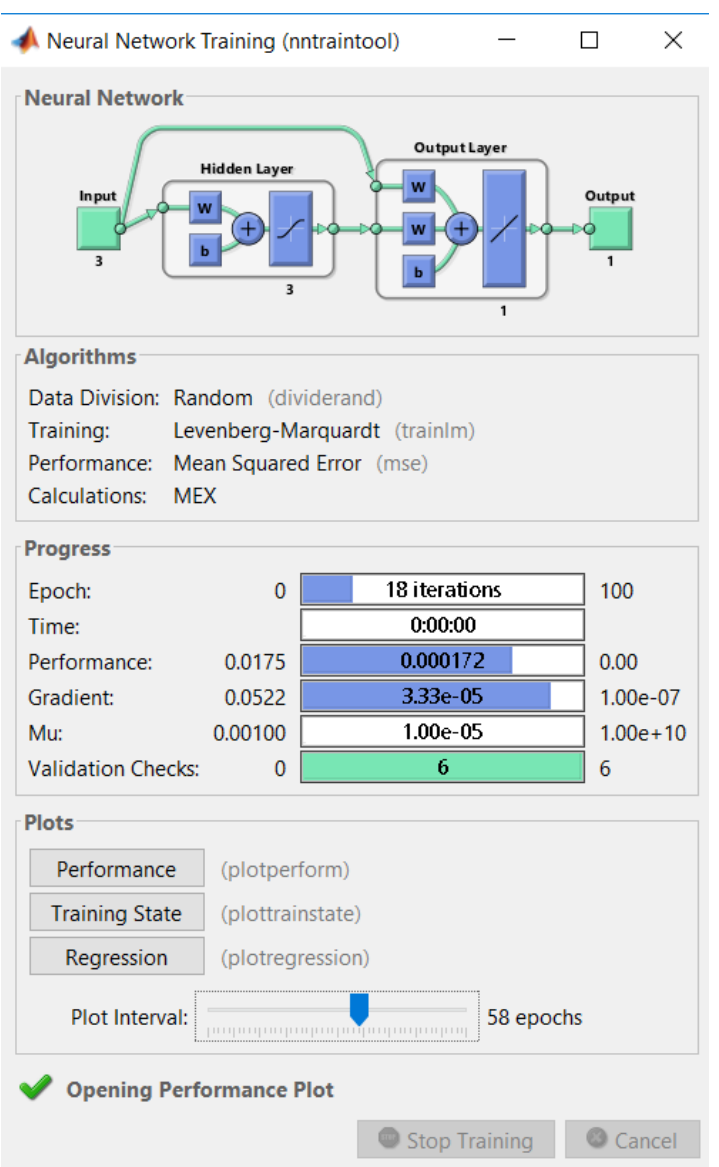

Fig.4. Neural network training report.

The results of the neural network training, verified on the validation set, have shown a $12 \%$ accuracy of fit of output parameters from the process in the function of the process parameters. Thus generated model consists of 3 independent variables and is represented in the graphic form in Fig. 5 and Fig. 6 for the roller contour radius $r=$ $0.8 \mathrm{~mm}$. The developed representation of the burnishing process has provided the reference model for the functional relationships occurring between the selected process parameters and effects of burnishing.

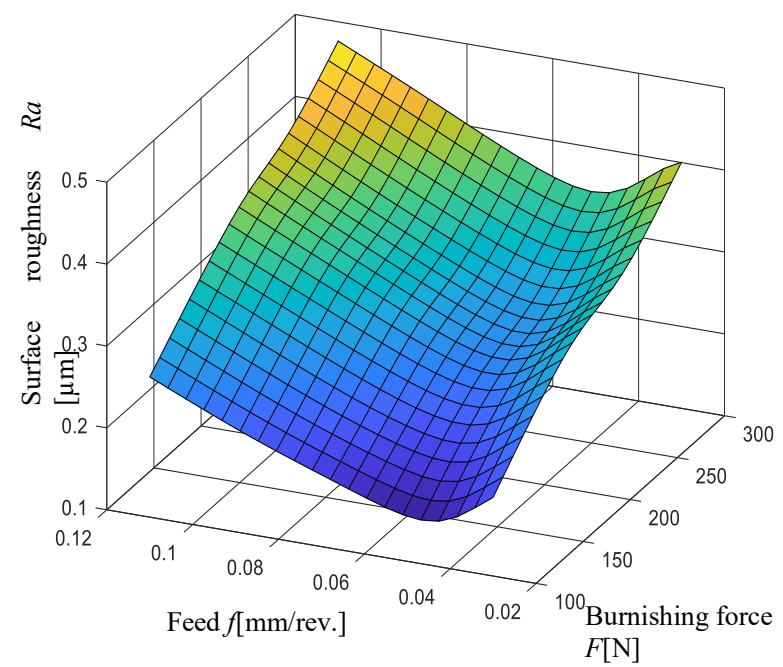

Fig.5. Graphic interpretation of relationship $R a=\mathrm{f}(F, f)$ for roller contour radius $r=0.8 \mathrm{~mm}$.

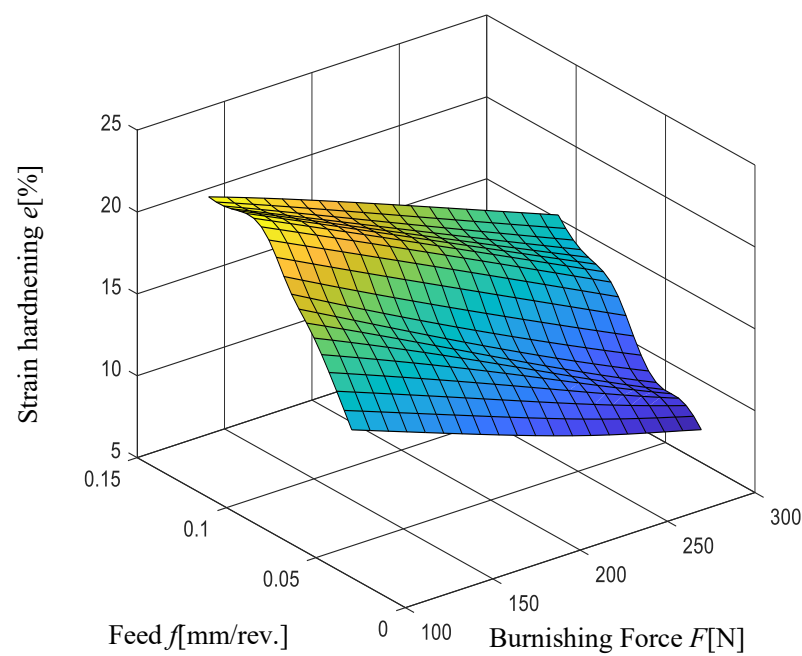

Fig.6. Graphic interpretation of relationship $e=f(F, f)$ for roller contour radius $\mathrm{r}=0.8 \mathrm{~mm}$

In the third stage of the algorithm application, the model reference relationship was used to determine burnishing parameters that produce the surface complying with the specified target surface roughness and strain hardening requirements. Obviously, both surface parameters must be developed simultaneously during a single process.

In order to account for the fact that the degree of strain hardening is the key surface characteristic imparted in the technological operation of roller burnishing, the algorithm assumed the maximal value of this parameter at $e=21 \%$, at the tolerance of $+1 \%$ $(e=20+1 \%)$, which corresponds to the maximum value recorded in the tests. The optimisation algorithm has 
generated a set of parameters which are expected to produce the target result (Table 1).

Table.1. Set of roller burnishing parameters ensuring maximal e and target surface roughness $R a$.

\begin{tabular}{|r|c|c|c|c|c|}
\hline No. & $\begin{array}{c}\text { Strain } \\
\text { hardening } \\
{[\%]}\end{array}$ & $R a[\mu \mathrm{m}]$ & $\begin{array}{c}\text { Roller } \\
\text { contour } \\
\text { radius } r \\
{[\mathrm{~mm}]}\end{array}$ & $\begin{array}{c}\text { Burnishing } \\
\text { force } F \\
{[\mathrm{~N}]}\end{array}$ & $\begin{array}{c}\text { Feed } f \\
{[\mathrm{~mm} / \mathrm{rev} .]}\end{array}$ \\
\hline 1 & 21.08 & 0.22 & 0.80 & 233.33 & 0.03 \\
\hline 2 & 21.83 & 0.25 & 0.80 & 255.56 & 0.03 \\
\hline 3 & 21.54 & 0.27 & 0.80 & 255.56 & 0.04 \\
\hline 4 & 21.01 & 0.29 & 0.80 & 255.56 & 0.05 \\
\hline 5 & 21.70 & 0.31 & 0.80 & 277.78 & 0.05 \\
\hline 6 & 21.61 & 0.34 & 0.80 & 300.00 & 0.06 \\
\hline 7 & 21.52 & 0.20 & 1.50 & 300.00 & 0.03 \\
\hline 8 & 21.09 & 0.24 & 1.50 & 300.00 & 0.05 \\
\hline 9 & 21.14 & 0.22 & 1.50 & 277.78 & 0.04 \\
\hline 10 & 21.53 & 0.22 & 1.50 & 300.00 & 0.04 \\
\hline 11 & 21.39 & 0.20 & 1.50 & 277.78 & 0.03 \\
\hline
\end{tabular}

The second technological objective of the analysed process is to produce minimal surface roughness $R a$ at a high degree of strain hardening. The simulation, performed by means of the model generated in the neural network for the subset of parameters from Table 1, has led to establishing the roller burnishing parameters that translate to the most significant decrease in the value of the surface roughness parameter in question, within the specified range of maximal strain hardening.

Further application of the developed algorithm for the determination of optimal process parameters depends on particular economic objectives and design constraints.

\section{Strategy 1:}

Maximise the strain hardening $e$ while minimising the surface roughness $R a$, considering that each value of $f$ form Table 1 is acceptable.

As a result, 2 equivalent sets of parameters are obtained: $r=1.5 \mathrm{~mm}, F=277.78 \mathrm{~N}, f=0.03 \mathrm{~mm} / \mathrm{rev}$. for $R a=0.2$ $\mu m$ and $e=21.39 \%$;

and

$r=1.5 \mathrm{~mm}, F=300 \mathrm{~N}, f=0.03 \mathrm{~mm} / \mathrm{rev}$. for $R a=0.2$ $\mu m$ and $e=21.52 \%$.

The feed value in Strategy 1 corresponds to half of the feed (in the subset of values burnishing feet) for maximal strain hardening, nevertheless, both $R a$ and $e$ are optimal regarding the surface quality.

\section{Strategy 2:}

Maximising the process efficiency (feed $f$ is proportional to process efficiency) while allowing for slight increase in the surface roughness $R a$ and maintaining the highest attainable strain hardening level. The strategy is realised by the two parameter sets:

$r=0.8 \mathrm{~mm}, F=300 \mathrm{~N}, f=0.06 \mathrm{~mm} / \mathrm{rev}$. for $R a=0.34$ $\mu m$ and $e=21.61 \%$;

and $r=1.5 \mathrm{~mm}, F=300 \mathrm{~N}, f=0.05 \mathrm{~mm} / \mathrm{rev}$. for $R a=0.24$ $\mu m$ and $e=21.09 \%$.

For the first parameter set the value of feed is twice as high as for Strategy 1, however, $R a=0.34 \mu \mathrm{m}$ which is $0.14 \mu \mathrm{m}$ more than the target value (minimal in the subset $0.2 \mu \mathrm{m})$.

Comparable efficiency is achieved when processing with the application of the second set of parameters: since feed $\mathrm{f}$ is lower by mere $0.01 \mathrm{~mm} / \mathrm{rev}$, and the difference in $\mathrm{Ra}$ is a mere $0.04 \mu \mathrm{m}$.

\section{Conclusion}

The described algorithm was developed in MATLAB $\mathbb{R}$ computation environment. It has been decided that programme will have a modular structure and will offer a high degree of configurability. The presented application contains the training module of the neural network representing a multidimensional technological process. The training module may be modified by the user in terms of the structure of the network and the goodness of fit of the modelled process' properties. The second module implements the trained neural network as a reference model for the optimisation process. The user defines the number of states for determining the minimal and maximal values of the output parameters, as well as the acceptable range for the output parameters. The module has been developed by means of Genetic Algorithm and Direct Search Toolbox MATLAB ${ }^{\circledR}$.

Module three serves as a visualisation tool for the developed model. Models with more than 2 variables are represented graphically for the 2 selected variables, while other values must be specified at a given level. The results can be also obtained in the form of tables.

As the presented example of determining the optimal parameters for the roller burnishing process shows, the neural network model employed to analyse the space of modifiable parameters is capable of designing a process exhibiting a two-fold increase in efficiency or a significant increase in the final product quality.

\section{References}

1. R. Noorossana, S. Davanloo Tajbakhsh, A. Saghaei, Int J Adv Manuf Technol, 40, 1227-1238 (2009)

2. T. Li, K. L. Hsieh, Qual Eng, 13, 11-18 (2000)

3. Z. T. Fu, W. Y. Yang, X. L. Wang, J. Leopold, 15th Cirp Conf. Model. Mach. Oper. (15th C. 31, 258263 (2015)

4. I. Danis, F. Monies, P. Lagarrigue, N. Wojtowicz, Int. J. Adv. Manuf. Technol. 84, 1801-1820 (2016)

5. D.C. Montgomery, Design and analysis of experiments 3rd edn. Wiley, New York, (1991)

6. R. H. Myers, D.C. Montgomery, Response surface methodology: process and product optimization using design experiments, 2nd edn. Wiley, New York, (2002)

7. M. Kulisz, I. Zagórski, A. Semeniuk, Appl. Comput. Sci. 12, 49-58 (2016) 
8. P. Kazemi, M. H. Khalid, J. Szlek, A. Mirtic, G. K. Reynolds, R. Jachowicz, A. Mendyk, Powder Technol. 301, 1252-1258 (2016)

9. P. Venkataraman, Applied Optimization with
Matlab Programing, Wiley \& Sons, New York, (2002)

1. 10. F. Ortiz, J.R. Simpson, J. J. Pignatiello, A. Heredia-Landgner, Qual Eng, 16, 11-18, (2004) 